\title{
A Bi-criteria Optimization Model for Adjusting the Decision Tree Parameters
}

\author{
Mohammad Azad ${ }^{1, *}$, Mikhail Moshkov ${ }^{2}$ \\ ${ }^{1}$ Jouf University \\ Department of Computer Science, College of Computer and Information Sciences \\ Sakaka 72441, Saudi Arabia \\ ${ }^{2}$ King Abdullah University of Science and Technology Computer, \\ Electrical and Mathematical Sciences \& Engineering Division \\ Thuwal 23955-6900, Saudi Arabia \\ *Corresponding author: mikhail.moshkov@kaust.edu.sa
}

\begin{abstract}
Decision trees play a very important role in knowledge representation because of its simplicity and selfexplanatory nature. We study the optimization of the parameters of the decision trees to find a shorter as well as more accurate decision tree. Since these two criteria are in conflict, we need to find a decision tree with suitable parameters that can be a trade off between two criteria. Hence, we design two algorithms to build a decision tree with a given threshold of the number of vertices based on the bi-criteria optimization technique. Then, we calculate the local and global misclassification rates for these trees. Our goal is to study the effect of changing the threshold for the bi-criteria optimization of the decision trees. We apply our algorithms to 13 decision tables from UCI Machine Learning Repository and recommend the suitable threshold that can give us more accurate decision trees with a reasonable number of vertices.
\end{abstract}

Keywords: Decision trees; Directed acyclic graph; Knowledge representation; Pareto optimal points; Misclassification; Bi-criteria optimization

\section{Introduction}

Decision trees are a very common and accepted model for the classifier (Azad et al., 2015, 2018; Breiman et al., 1984). They can also be used to find the patterns and represent knowledge from the data (Alsolami et al., 2020; Azad et al., 2019; Breiman et al., 1984). Furthermore, it can be used as algorithms to solve problems (Moshkov, 2005; Rokach and Maimon, 2008). The problems considered in this paper are similar to the problems in medical diagnosis (Podgorelec et al., 2002), image analysis (Mehmood et al., 2018; Sarwar et al., 2018), pattern recognition (Yahyaoui et al., 2020) etc. We choose decision tree in this paper because of its easy to use nature and easily comprehensible and explainable by humans as well as directly exchangeable to production rules (Almuallim et al., 2002). Optimizing the parameters of decision tree can be extremely useful based on the context: minimizing depth or average depth can be equivalent to minimizing the time complexity of decision trees, minimizing number of vertices or number of leaf/non-leaf vertices can be equivalent to minimizing the space complexity of decision trees and minimizing the number of misclassification can be equivalent to maximizing the accuracy of decision trees etc. In this paper, we consider optimizing three parameters of decision trees: number of vertices and misclassification rates (global and local). 
It is important to consider not only optimizing single criteria but also optimizing two criteria at the same time which is popularly known as the bi-criteria optimization (BCO). BCO is a part of a broader class of optimization problems named as multi-criteria optimization (MCO). In the literature, we can find it in various names e.g., multiple objective discrete optimization, vector optimization, multiple objective combinatorial optimization, multi-attribute optimization, multiple criteria decision making, Pareto optimization (Chang, 2015; Ehrgott and Gandibleux, 2000; Kouvelis and Sayın, 2006), etc. Let us see some examples in which MCO has been applied successfully in many real world scientific and engineering problems where we choose optimal solutions as a trade-offs between two or more conflicting objectives. One of the examples is the maximizing performance while minimizing fuel consumption and emission of pollutants of a vehicle (Chang, 2015). Another example is the searching for new remedial drugs in the medical field: maximizing strength of the drug while minimizing synthesis costs and unwanted side effects (Emmerich Michael T. M., 2018; Rosenthal and Borschbach, 2017; van der Horst et al., 2012). There are many other examples that brought the attention of the research community: the minimization of different types of error rates in machine learning (false positives, false negatives) (Wang et al., 2015; Yevseyeva et al., 2013), the optimization of delivery costs and inventory costs in logistics (Geiger and Sevaux, 2011), the optimization of building designs with respect to health, energy efficiency, and cost criteria (Hopfe et al., 2012). In this paper, we consider BCO relative to the number of vertices and misclassification rate (either global or local).

In $\mathrm{BCO}$ and $\mathrm{MCO}$, the objective functions are said to be in conflict and the goal is to find a solution that is optimal in some sense. The solutions that can not be improved in one objective without deteriorating the performance in any other objective are called Pareto optimal or nondominated solutions. They are named after Italian economist Vilfredo Pareto (de Weck, 2004).

In this paper, we build the set of Pareto optimal points (POPs) for BCO relative to the number of vertices and misclassification rate (either g lobal or 1 ocal). It is i mportant to find a de cision tree with a reasonable number of vertices to make it understandable and with a reasonable misclassification rate (either global or local) to extract accurate knowledge from it. In (Azad et al., 2019), we mentioned that we should focus not only on the global misclassification rate but also on the local misclassification rate because the misclassifications may not be equally distributed among the leaf $v$ ertices. Besides, it may happen that the fraction of misclassifications can be high for some of the leaf vertices.

Previously, we created a dynamic programming (Bellman, 1954; Tian et al., 2018) approach to BCO of decision trees for decision tables relative to two parameters (AbouEisha et al., 2019). The idea is to first build a directed acyclic graph (DAG) then, from DAG (using special algorithm mentioned in (AbouEisha et al., 2019)) build the set of POPs. After that, we can derive decision trees which parameter values will be in accordance with the desired POP. Note that, the time complexity of such process depends on the size of the considered table and, in the worst case, it is exponential. Therefore, from the practical point of view, we can work from small to medium sized decision tables.

In (Azad et al., 2019), the designed algorithms based on BCO are pertinent to medium-sized decision tables with categorical features only and, sometimes, the number of vertices in the trees is very large. In (Azad, 2020b), instead of conventional decision trees, we study trees based on binary splits similar to CART (Classification and Regression Tree) (Breiman et al., 1984). We call such trees as BS-trees. BStrees use binary splits instead of the initial features. The standard CART tree uses in each internal vertex the best split among all features. A BS-tree can use in each internal vertex the best split for an arbitrary feature. It extends essentially the set of decision trees under consideration. In (Azad, 2020a), two algorithms $(G-19$ and $L-19)$ have been designed that build decision trees with at most 19 vertices (at most 10 leaves and at most nine internal vertices). The choice of 19 vertices was inspired by (AbouEisha et al., 2019).

In this paper, we try to understand the behavior of the parameters of the extracted decision trees if we change the value of the threshold from 19 to some other values like 21, 23, etc. Furthermore, we extend the algorithms $G-19$ and $L-19$ to algorithms $G_{\alpha}$ and $L_{\alpha}$ where $\alpha$ is a threshold that can take values $13, \ldots, 35$ with step 2 . We apply the considered algorithms to 13 data sets from the UCI Machine Learning Repository (Dua and Graff, 2017), and study three parameters of the created trees (number of 
Table 1. Example of a decision table $S_{0}$

$S_{0}=$\begin{tabular}{|cc|c|}
\hline$f_{1}$ & $f_{2}$ & \\
\hline 0 & 0 & 2 \\
0 & 1 & 2 \\
1 & 0 & 3 \\
1 & 1 & 1 \\
\hline
\end{tabular}

nodes, global misclassification rates, local misclassification rates). For each threshold value, we took the average value of three parameters based on the 13 tables. Based on the achieved results, we can easily adjust the number of vertices and misclassification error rates of decision trees based on the considered bi-criteria optimization model. Moreover, we recommend the range of threshold values between 15 to 21 with step 2 which can be valuable for significant analysis of decision trees for knowledge representation.

The main contribution of the paper are (i) creation of two algorithms $\left(G_{\alpha}\right.$ and $\left.L_{\alpha}\right)$, (ii) performing the experiments on the state-of-art data sets from UCI ML Repository, and (iii) understanding the trade-off between two conflicting objective of number of nodes vs. misclassification rates when we fix the number of nodes to a threshold.

We arrange the remaining of the manuscript as follows. First, we explain the detailed methodology in Section 2. Then, we discuss the output of the experiments with data sets from the UCI Machine Learning Repository (Dua and Graff, 2017) in Sect. 3. Finally, Sect. 4 contains concise conclusion.

\section{Detailed Methodology}

Many researchers have spent a great amount of research of greedy algorithms (Azad et al., 2013; Boryczka and Kozak, 2009; Breiman et al., 1984; Chai et al., 1996; Heath et al., 1993; Quinlan, 2014) for building non-optimal decision trees. These algorithms are faster but can't guarantee the optimal parameters of decision trees and did not consider BCO. In addition, some other researchers have studied the exhaustive algorithms for building optimal decision trees (Garey, 1972; Schumacher and Sevcik, 1976). But they did not consider BCO problem of the optimal decision tree parameters. However, only few have concentrated the study on dynamic programming for bi-criteria (and multi-criteria) optimization of decision trees (AbouEisha et al., 2019; Alsolami et al., 2020; Azad, 2018).

In this section, we describe an expansion of dynamic programming, which gives all possible decision trees under consideration. The sequence we follow is first, we describe the preliminary ideas of decision tables, decision trees and building of directed acyclic graph (DAG) then we demonstrate the properties of BS-trees, later we explain the BCO, and finally, we explain two algorithms $G_{\alpha}$ and $L_{\alpha}$.

\subsection{Preliminaries of Decision Tables and Decision Trees}

There are various types of decision tables: usual decision tables (AbouEisha et al., 2019), decision tables with multiple decisions (Alsolami et al., 2020; Azad, 2018) etc. In this paper, we consider usual decision table i.e., conventional single decision table where each row is attached with a single decision. We represent such decisions as nonnegative integers. Columns of this table are labeled with conditional attributes. Rows of the table are distinct. An example of a decision table $S_{0}$ is presented in Table 1 . The table $S_{0}$ consists of 4 rows and 2 attributes $\left(f_{1}\right.$ and $f_{2}$ ). We attach decisions $2,2,3,1$ corresponding to the row numbers $1,2,3$ and 4 respectively.

A decision tree for a decision table is a finite rooted directed tree. In such trees, there are two types of vertices: non-leaf vertices and leaf vertices. We label the non-leaf vertices with the conditional attributes from the decision tables and the leaf vertices with nonnegative integers (e.g., decisions attached with rows). We label outgoing edges in each non-leaf vertex with pairwise nonnegative integers (attribute values). We depict an example of an exact decision tree for the decision table $S_{0}$ in Fig. 1.

Now, we will describe how to create decision trees for the given decision table using an expansion of dynamic programming. Previously, we described such a process in many publications (AbouEisha et al., 2019; Aldilaijan et al., 2019; Alsolami et al., 2020; Azad, 2018; Azad and Moshkov, 2017). The 


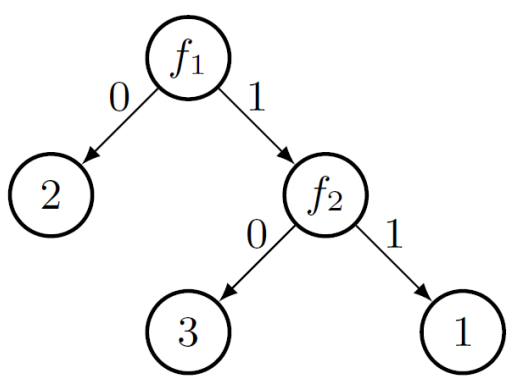

Fig. 1. A decision tree for the table $S_{0}$

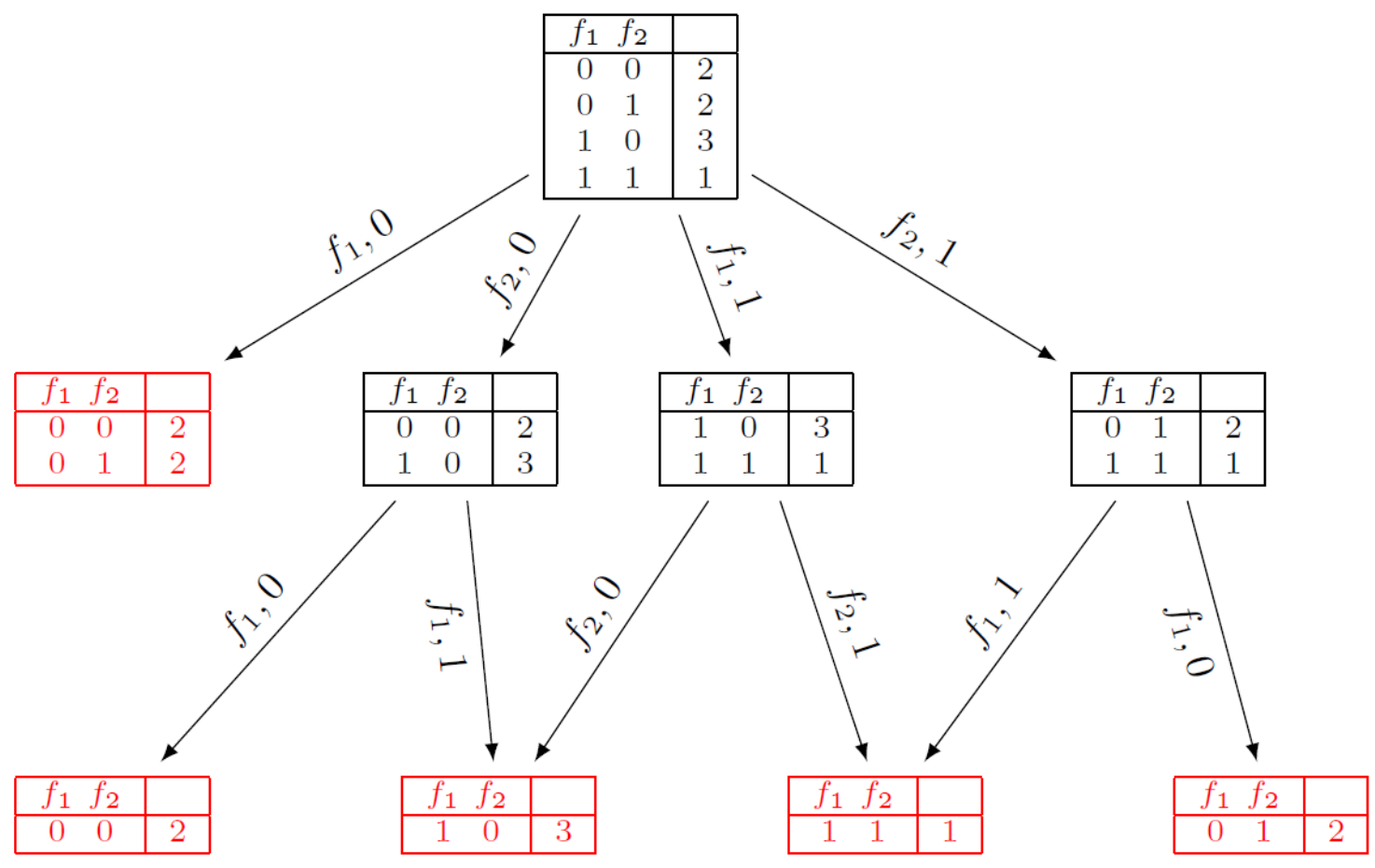

Fig. 2. The directed acyclic graph for the decision table $S_{0}$

idea is to build a directed acyclic graph (DAG) based on the expansion of dynamic programming. For example, a decision table $S_{0}$ is shown in Table 1 and corresponding DAG in Fig. 2.

After building the DAG, we can extract all decision trees for the decision table under consideration. The DAG in Fig. 2 describes two decision trees (illustrated in Fig. 3) for the decision table $S_{0}$. Even though the time complexity of the building of the DAG is exponential in the worst case subject to the size of the table, it is still possible to use this method for small to medium sized decision tables (AbouEisha et al., 2019; Alsolami et al., 2020; Azad, 2018).

There are many ways to split or partition the tables into subtables. The splits based on each value are considered in paper (Azad et al., 2019) which are applicable for categorical attributes only. In this paper, we consider the binary split similar to (Azad et al., 2015, 2018; Breiman et al., 1984). The advantage of binary split in comparison to each value split is that it can be applied for both categorical and numerical attributes.

\subsection{Binary Split Trees (BS-Trees)}

Being motivated by CART algorithm, we consider BS-trees similar to trees in CART. The difference is that a BS-tree uses in each non-leaf vertex the optimal partition for an arbitrary attribute, whereas, the 

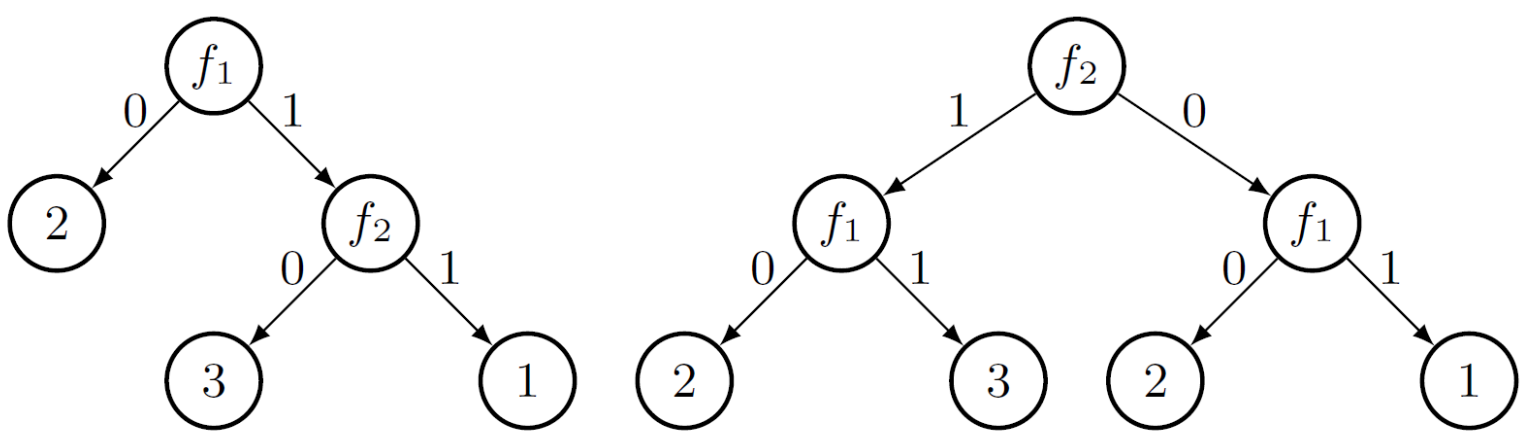

Fig. 3. Two possible decision trees for the table $S_{0}$

standard CART tree uses in each non-leaf vertex the optimal partition among all attributes.

Given a decision table $S$ containing $f_{1}, \ldots, f_{n}$ attributes (either numerical or categorical), we convert each attribute $f_{i}(1 \leq i \leq n)$ into binary attribute (as in CART) using the following procedure (we have two scenarios depending on the type of attributes):

1. Categorical attribute: Let $A$ be the set of values of the categorical attribute $f_{i}$. Then $A$ is partitioned into two nonempty subsets $A_{1}$ and $A_{0}$. If the value of attribute $f_{i}$ belongs to $A_{0}$ then we make the binary split $b s$ equal to 0 . Similarly, if the value of attribute $f_{i}$ belongs to $A_{1}$ then we make the binary split $b s$ equal to 1 . We need to take splits for all possible partitions of $A$.

2. Numerical attribute: Let us compare the values of the numerical attribute $f_{i}$ with a real-value threshold $\psi$. The split $b s$ is considered as 0 if $f_{i}$ 's value is smaller than $\psi$, and 1 otherwise. We consider splits for all possible thresholds $\psi$.

As a result of the partition $b s$, two subtables $S_{(b s=0)}$ and $S_{(b s=1)}$ are obtained. This is the main idea behind the binary partitioning.

In literature, "entropy" (Quinlan, 1986, 2014), "gini index" (AbouEisha et al., 2019) and "abs" (Alsolami et al., 2020; Azad, 2020b; Azad et al., 2015, 2018) were used as uncertainty measures. In this paper, we apply "abs" as the uncertainty measure. We get two subtables $S_{(b s=0)}$ and $S_{(b s=1)}$ by the partition $b s$ for the table $S$. We define the notion of impurity function $I(S, b s)$ as the weighted sum of uncertainties of these subtables i.e., $S_{(b s=0)}$ and $S_{(b s=1)}$ (the weights are based on the proportion of the total objects (rows) in the corresponding subtables). Now, we need to find the partition $b s$ for which the value of $I(S, b s)$ is minimum after applying the partition $b s$ for the attribute $f_{i}$ in $S$. Then we call such partition as the best partition for the attribute $f_{i}$.

We consider BS-trees where every leaf vertex is labeled by a non-negative integer. We label every non-leaf vertex by a partition based on one of the attributes. Also, two arcs are going out from this nonleaf vertex (one is labeled with 0 and another with 1). Let us consider a decision tree $\delta$ and any vertex $v$ of it. Then, we can map a subtable $S(\delta, v)$ for the given table $S$ for each vertex $v$. This subtable $S(\delta, v)$ consists of all objects (rows) of the given table $S$ for which the action of the decision tree proceeds through the vertex $v$. We build our tree based on the following assumptions:

1. For each non-leaf vertex $v, S(\delta, v)$ contains the objects (rows) that have different decisions. Furthermore, the vertex $v$ is labeled by an optimal split for a non-constant attribute $f_{i}$ on $S(\delta, v)$.

2. For each leaf vertex $v$, the vertex is labeled with a most frequent decision e.g., a decision which is bound with the largest amount of objects (rows) from $S(\delta, v)$.

We examine the following three parameters of the tree $\delta$ (Azad et al., 2019): 


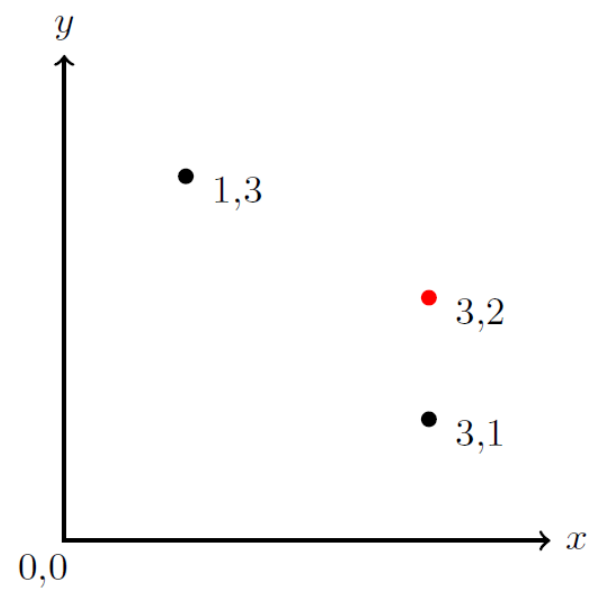

Fig. 4. Example of POPs. The black points are POPs and the red point is not POP

1. The number of vertices: we denote it by $N$. It is the total number of vertices including leaf and non-leaf vertices.

2. The global misclassification rate: we denote it by $m e^{G}$. We calculate the number of misclassifications of $\delta$ on $S$ and divide it by the number of rows in $S$.

3. The local misclassification rate: we denote it by $m e^{L}$. For each leaf vertex of $\delta$, we calculate the ratio of the number of misclassifications among rows accepted by this vertex to the number of all rows accepted by the vertex. Then, we take the maximum of the obtained values among all leaf vertices of $\delta$.

One can show that the global misclassification rate is at most the local misclassification rate.

\subsection{Bi-criteria Optimization (BCO) and Pareto Fronts}

When we consider BCO in the presence of trade-offs between two objectives that may be in conflict, we need to find optimal solutions (pair of values of two o bjectives) (Chang, 2015). For example, if we increase the number of vertices in the trees then the misclassification rate (either global or local) decreases and vice versa. A solution is called nondominated if one objective functions can not be improved in value without degrading another objective function value. We call such solutions as Pareto optimal points (POPs) (Chang, 2015).

Let us consider a point $\left(i_{1}, j_{1}\right)$ in $D$ (where $D$ is a finite set of points in a two-dimensional space). This point is called a Pareto optimal point (POP) for $D$ if we do not find any other point $\left(i_{2}, j_{2}\right)$ in $D$ where $i_{2} \leq i_{1}$ and $j_{2} \leq j_{1}$ such that $\left(i_{1}, j_{1}\right) \neq\left(i_{2}, j_{2}\right)$ (AbouEisha et al., 2019; Alsolami et al., 2020; Azad, 2018). We can see a simple example in Fig. 4 where the black points are POPs and the red point is not POP. The collection of POPs for a particular problem is called the Pareto front of the problem.

In this paper, our goal is to minimize both number of vertices and misclassification rate (either global or local) of the trees at the same time and they are in conflict. The algorithm $\mathcal{A}_{\mathrm{POPs}}$ (AbouEisha et al., 2019; Alsolami et al., 2020; Azad, 2018) will be used to find all POPs for this BCO relative to the number of vertices and misclassification rate (either global or local).

\subsection{Two Algorithms for the building of BS-Trees}

In this section, we describe how to build the BS-Trees.

The first step is to c reate a directed a cyclic $\mathrm{g}$ raph (DAG) for the given decision table, vertices of which are some subtables of the considered decision table. For this purpose, we use the algorithm $\mathcal{A}_{1}$ from (AbouEisha et al., 2019; Alsolami et al., 2020; Azad, 2018; Azad et al., 2015). The algorithm $\mathcal{A}_{1}$ works as follows: it first partitions the main table into subtables based on the all possible combination of non-constant attributes and their values. This process of partitioning will continue to the newly generated 


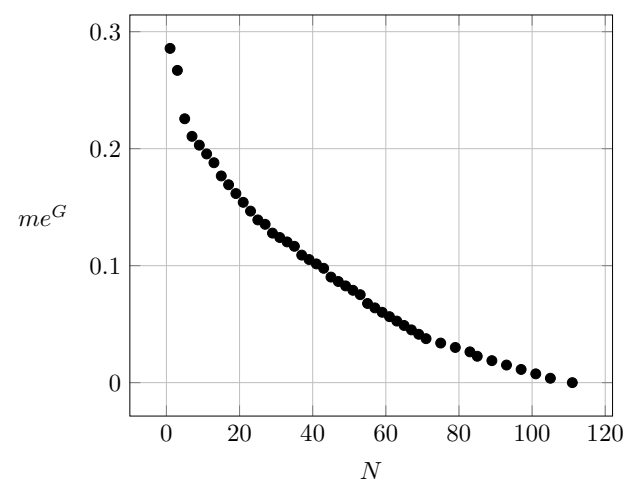

(a) S2, $N$ and $m e^{G}$

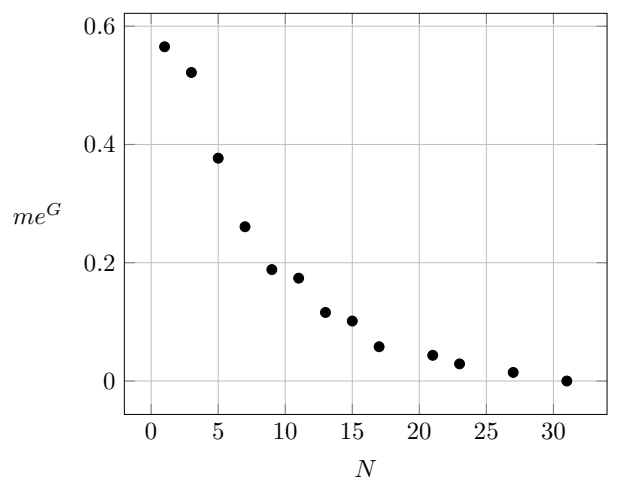

(c) $\mathrm{S} 4, N$ and $m e^{G}$

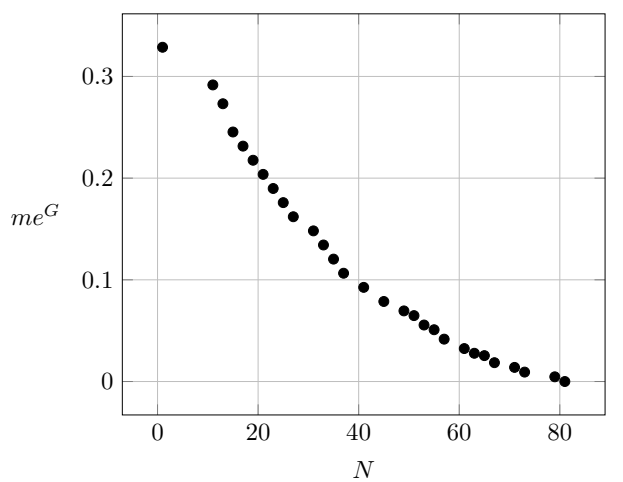

(e) S6, $N$ and $m e^{G}$

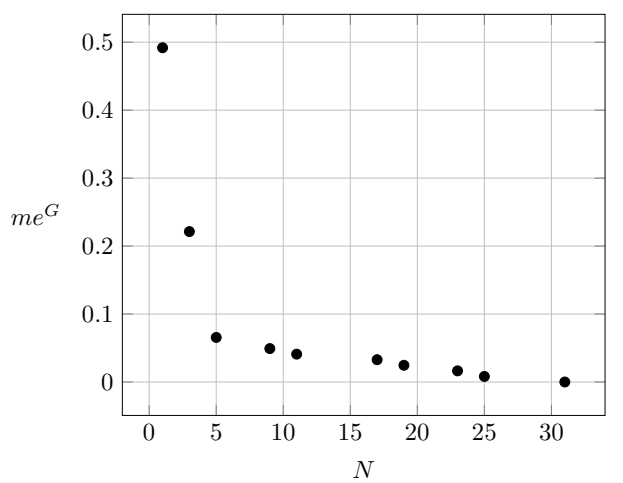

(g) S8, $N$ and $m e^{G}$

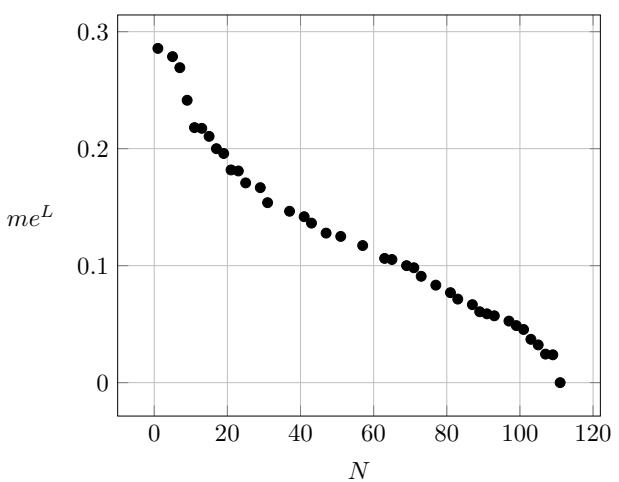

(b) S2, $N$ and $m e^{L}$

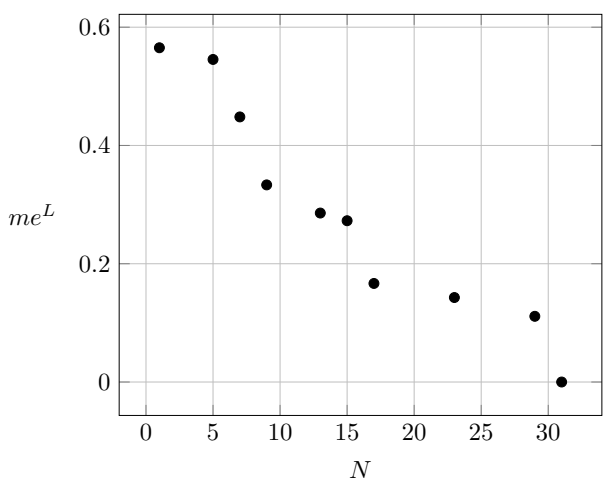

(d) S4, $N$ and $m e^{L}$

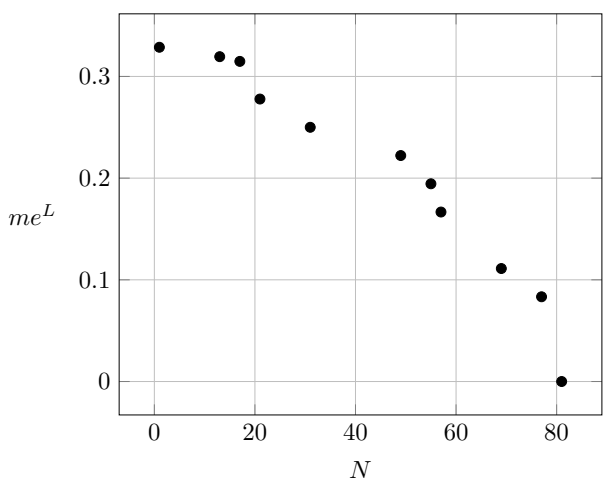

(f) S6, $N$ and $m e^{L}$

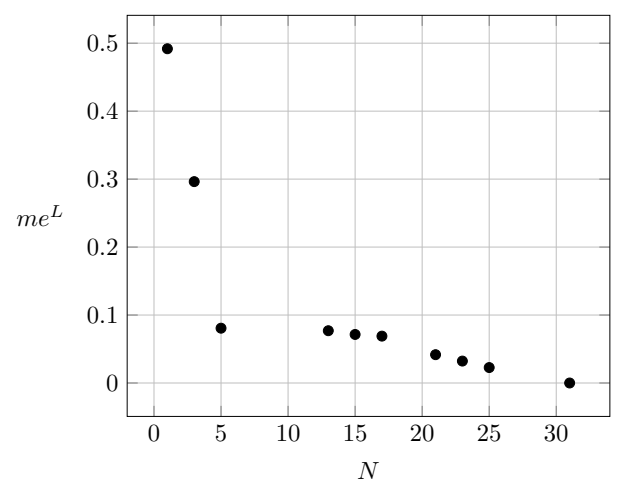

(h) S8, $N$ and $m e^{L}$

Fig. 5. Sets of Pareto optimal points for decision tables S2, S4, S6, and S8 (see Table 2) for pairs of parameters $N, m e^{G}$ and $N, m e^{L}$ 
subtables until the newly generated subtables contain the same decision for each row. Also note that we generate a single instance of the subtables in the graph to maintain the property of the DAG. As an example, we can find the DAG for the decision table $S_{0}$ in Fig. 2.

As the next step, we use the algorithm $\mathcal{A}_{\mathrm{POPs}}$ from (AbouEisha et al., 2019; Alsolami et al., 2020; Azad, 2018; Azad et al., 2015). The output of this algorithm is the Pareto front - the set of all POPs (Pareto optimal points) for BCO of BS-trees relative to two criteria for each vertex (subtable) of the created DAG. The algorithm $\mathcal{A}_{\mathrm{POPs}}$ works as follows: it traverses through smaller subtables to bigger subtables using the created DAG in a bottom up fashion. It begins with nodes that are labeled with subtables containing the same decision for each row until it reaches the root node which is labeled with the main table. At each node, it creates the set of POPs for that node based on the specific formula to combine POPs from the child nodes and remove those points which are not POPs due to this combination. This process continues and at the end, we will get the set of POPs at the root node for the main table. Next, for each POP, we can extract a tree with parameter values equal to the coordinates of this point.

In this paper, we use the algorithm $\mathcal{A}_{\mathrm{POPs}}$ to solve two $\mathrm{BCO}$ problems: relative to the parameters $N$ and $m e^{G}$ (see examples in Fig. 5 (a), (c), (e), (g)) and relative to the parameters $N$ and $m e^{L}$ (see examples in Fig. 5 (b), (d), (f), (h)).

We now describe two algorithms to design decision trees using the algorithm $\mathcal{A}_{\mathrm{POPs}}$. We assume that the DAG for the considered decision table is already created by the algorithm $\mathcal{A}_{1}$. Note that the time complexity of the algorithms $\mathcal{A}_{1}$ and $\mathcal{A}_{\mathrm{POPs}}$ is exponential in the worst case subject to the size of the decision tables. Therefore, in the worst case, the following two algorithms have exponential time complexity as well.

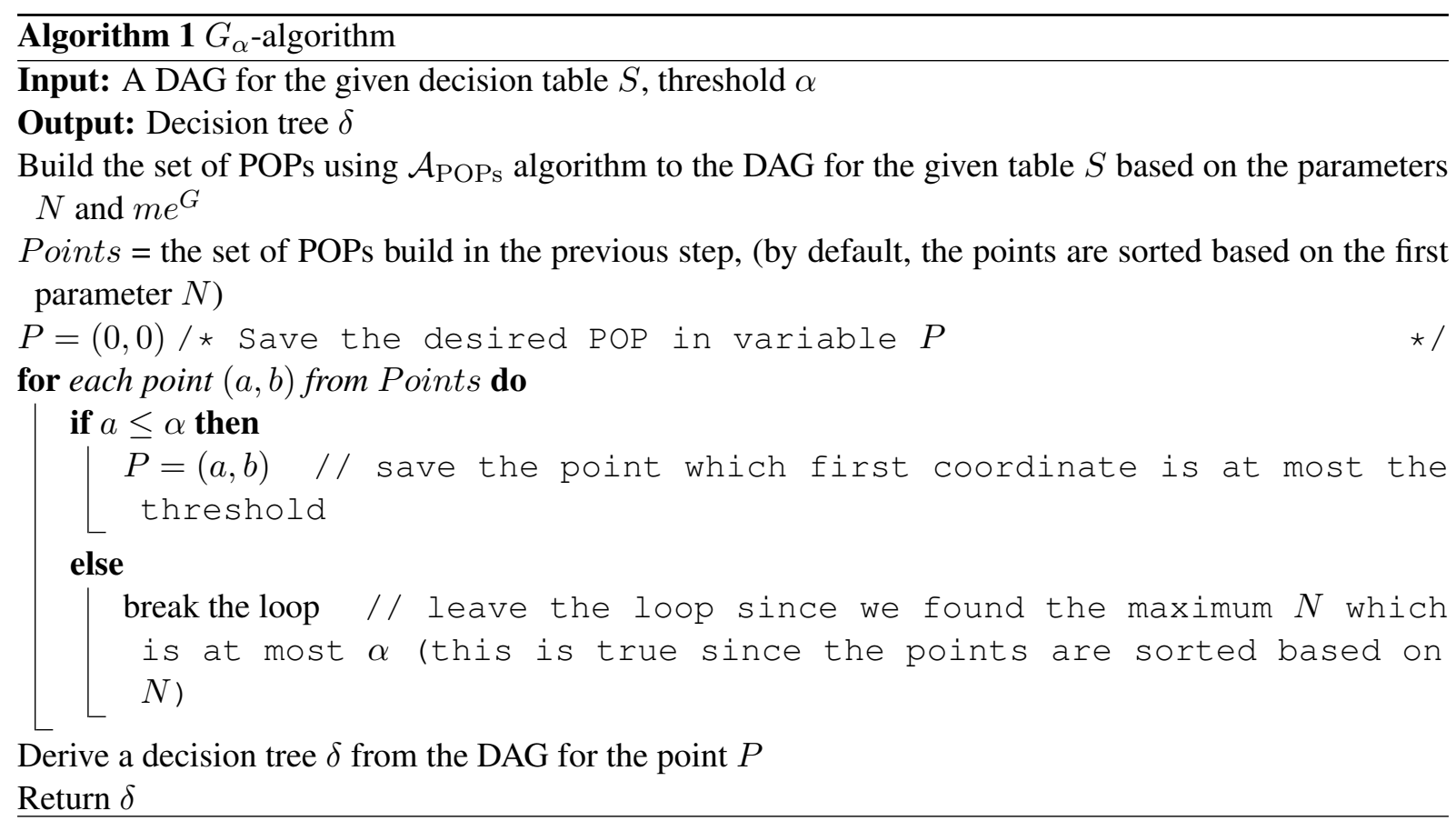

\subsection{1 $G_{\alpha}$-algorithm}

We apply the algorithm $\mathcal{A}_{\mathrm{POPs}}$ to the DAG for the decision table $S$. The algorithm's output is the set of POPs for the BCO of BS-trees for parameters $N$ and $m e^{G}$. We choose a POP with the maximum value of the parameter $N$ which is at most $\alpha$. After that, we extract a decision tree $\delta$, for which the parameters $N$ and $m e^{G}$ are equal to the coordinates of this POP. The tree $\delta$ is the output of $G_{\alpha}$-algorithm. We describe the pseudo code of $G_{\alpha}$-algorithm in Algorithm 1. 
Table 2. Description of the decision tables used in the experiments

\begin{tabular}{llrr}
\hline Table & Name & Rows & Attributes \\
\hline$S 1$ & BALANCE-SCALE & 625 & 5 \\
$S 2$ & BREAST-CANCER & 266 & 10 \\
$S 3$ & CARS & 1728 & 7 \\
$S 4$ & HAYES-ROTH-DATA & 69 & 5 \\
$S 5$ & HOUSE-VOTES-84 & 279 & 17 \\
$S 6$ & MONKS-2-TEST & 432 & 6 \\
$S 7$ & MONKS-2-TRAIN & 169 & 5 \\
$S 8$ & MONKS-3-TRAIN & 122 & 6 \\
$S 9$ & LYMPHOGRAPHY & 148 & 19 \\
$S 10$ & NURSERY & 12960 & 9 \\
$S 11$ & SPECT-TEST & 169 & 23 \\
$S 12$ & TIC-TAC-TOE & 958 & 10 \\
$S 13$ & ZOO-DATA & 59 & 17 \\
\hline
\end{tabular}

\subsection{2 $L_{\alpha}$-algorithm}

This algorithm is same as $G_{\alpha}$ except that we create the set of POPs for the BCO of BS-trees for parameters $N$ and $m e^{L}$. We choose a POP with the maximum value of the parameter $N$ which is at most $\alpha$. After that, we extract a decision tree $\delta$, for which the parameters $N$ and $m e^{L}$ are equal to the coordinates of this POP. The tree $\delta$ is the output of $L_{\alpha}$-algorithm.

\section{Results of Experiments}

We have used 13 decision tables from the UCI Machine Learning Repository (Dua and Graff, 2017) for the experiments (described in Table 2).

We applied $G_{\alpha}$ and $L_{\alpha}$ algorithms to each of these tables for the threshold $\alpha=13, \ldots, 35$ with step 2 and found values of the parameters $N, m e^{G}$, and $m e^{L}$ for the created decision trees. Table 3 describes the experimental results for the four thresholds 21, 23, 31 and 33 ('Avg' means average values of the parameters).

We can see from the results that the $L_{\alpha}$-algorithm in comparison to $G_{\alpha}$-algorithm gives us smaller values of the parameter $m e^{L}$. Similarly, the $G_{\alpha}$-algorithm in comparison to $L_{\alpha}$-algorithm gives us the smaller values of the parameter $m e^{G}$. Furthermore, the general pattern is that the $N$ parameter is decreasing, whereas, $m e^{G}$ and $m e^{L}$ parameters are increasing with the decrease of the value of the threshold. Hence, we can easily adjust the number of vertices to get the desired misclassification rates using this optimization model.

We took the averages of the parameters over the 12 thresholds which are shown in Table 4. Here, $N_{A}$ denotes the average of the parameter $N, m e_{A}^{G}$ denotes the average of the parameter $m e^{G}$ and $m e_{A}^{L}$ denotes the average of the parameter $m e^{L}$. Below, we analyze the changes of the average values of the parameters for the decrease of the threshold.

We can see the changes of the average values of $N, m e^{G}$ and $m e^{L}$ with the decrease of the threshold from 35 to 13 (by step 2) for both $G_{\alpha}$ and $L_{\alpha}$ algorithms in Table 5. If we investigate the changes, we do not find any threshold which is much better than others. In general, if we decrease the threshold, the average value of the parameter $N$ decreases and the average values of the parameters $m e^{L}$ and $m e^{G}$ increase step by step. Rather than finding a single threshold, we may consider a range of thresholds which can be enough interesting.

- Let us first consider the $m e_{A}^{G}$ parameter of the $G_{\alpha}$-algorithm. If we decrease the threshold, initially the average value is increasing slowly and at the threshold 15, it greatly increases compared to the previous threshold 17.

- Next let us consider the $m e_{A}^{L}$ parameter of the $G_{\alpha}$-algorithm. If we decrease the threshold, the behavior of the average value is not stable. Sometimes it is increasing then it decreases, again it is 
Table 3. Different thresholds and corresponding results

\begin{tabular}{lcccccc}
\hline Table & \multicolumn{3}{c}{$G_{21}$ algorithm } & \multicolumn{3}{c}{$L_{21}$ algorithm } \\
& \multicolumn{2}{c}{$N$} & $m e^{G}$ & $m e^{L}$ & \multicolumn{3}{c}{$N$} & $m e^{G}$ & $m e^{L}$ \\
\hline$S 1$ & 21 & 0.18 & 0.46 & 21 & 0.20 & 0.31 \\
$S 2$ & 21 & 0.15 & 0.28 & 21 & 0.17 & 0.18 \\
$S 3$ & 21 & 0.09 & 0.39 & 21 & 0.12 & 0.22 \\
$S 4$ & 21 & 0.04 & 0.17 & 17 & 0.06 & 0.17 \\
$S 5$ & 19 & 0.02 & 0.02 & 19 & 0.02 & 0.02 \\
$S 6$ & 21 & 0.05 & 0.20 & 21 & 0.06 & 0.10 \\
$S 7$ & 21 & 0.20 & 0.33 & 21 & 0.25 & 0.28 \\
$S 8$ & 21 & 0.18 & 0.29 & 19 & 0.21 & 0.25 \\
$S 9$ & 19 & 0.02 & 0.25 & 21 & 0.02 & 0.04 \\
$S 10$ & 21 & 0.10 & 0.32 & 21 & 0.11 & 0.20 \\
$S 11$ & 21 & 0.01 & 0.06 & 21 & 0.02 & 0.02 \\
$S 12$ & 21 & 0.16 & 0.39 & 21 & 0.19 & 0.24 \\
$S 13$ & 17 & 0.00 & 0.00 & 17 & 0.00 & 0.0 \\
\hline Avg & 20.38 & 0.09 & 0.24 & 20.08 & 0.11 & 0.16 \\
\hline \multicolumn{5}{c}{ Threshold 21} \\
\end{tabular}

\begin{tabular}{|c|c|c|c|c|c|c|}
\hline \multirow{2}{*}{$\begin{array}{l}\text { Table } \\
S 1\end{array}$} & \multicolumn{3}{|c|}{$\begin{array}{l}G_{23} \text { algorithm } \\
N m e^{G} m e^{L}\end{array}$} & \multicolumn{3}{|c|}{$\begin{array}{l}L_{23} \text { algorithm } \\
N m e^{G} m e^{L}\end{array}$} \\
\hline & 23.00 & 0.17 & 0.33 & 23.00 & 0.22 & 0.25 \\
\hline$S 2$ & 23.00 & 0.15 & 0.28 & 23.00 & 0.17 & 0.18 \\
\hline$S 3$ & 23.00 & 0.08 & 0.40 & 21.00 & 0.12 & 0.22 \\
\hline$S 4$ & 23.00 & 0.03 & 0.17 & 23.00 & 0.04 & 0.14 \\
\hline$S 5$ & 19.00 & 0.02 & 0.03 & 19.00 & 0.02 & 0.02 \\
\hline$S 6$ & 23.00 & 0.04 & 0.20 & 23.00 & 0.07 & 0.09 \\
\hline$S 7$ & 23.00 & 0.19 & 0.33 & 21.00 & 0.25 & 0.28 \\
\hline$S 8$ & 23.00 & 0.16 & 0.32 & 23.00 & 0.21 & 0.24 \\
\hline$S 9$ & 23.00 & 0.02 & 0.08 & 23.00 & 0.02 & 0.03 \\
\hline$S 10$ & 23.00 & 0.10 & 0.34 & 21.00 & 0.11 & 0.20 \\
\hline$S 11$ & 21.00 & 0.01 & 0.13 & 23.00 & 0.01 & 0.02 \\
\hline$S 12$ & 23.00 & 0.15 & 0.39 & 23.00 & 0.19 & 0.23 \\
\hline$S 13$ & 17.00 & 0.00 & 0.00 & 17.00 & 0.00 & 0.00 \\
\hline Avg & 22.08 & 0.09 & 0.23 & 21.77 & 0.11 & 0.15 \\
\hline \multicolumn{7}{|c|}{ Threshold 23} \\
\hline Table & \multicolumn{3}{|c|}{$\begin{array}{l}G_{33} \text { algorithm } \\
N m e^{G} m e^{L}\end{array}$} & \multicolumn{3}{|c|}{$\begin{array}{l}L_{33} \text { algorithm } \\
N m e^{G} m e^{L}\end{array}$} \\
\hline$S 1$ & 33.00 & 0.15 & 0.50 & 23.00 & 0.22 & 0.25 \\
\hline$S 2$ & 33.00 & 0.12 & 0.33 & 31.00 & 0.14 & 0.15 \\
\hline$S 3$ & 33.00 & 0.06 & 0.34 & 29.00 & 0.14 & 0.21 \\
\hline$S 4$ & 31.00 & 0.00 & 0.00 & 31.00 & 0.00 & 0.00 \\
\hline$S 5$ & 31.00 & 0.01 & 0.02 & 33.00 & 0.01 & 0.01 \\
\hline$S 6$ & 33.00 & 0.01 & 0.08 & 33.00 & 0.01 & 0.03 \\
\hline$S 7$ & 33.00 & 0.13 & 0.33 & 31.00 & 0.22 & 0.25 \\
\hline$S 8$ & 33.00 & 0.10 & 0.32 & 33.00 & 0.16 & 0.22 \\
\hline$S 9$ & 31.00 & 0.00 & 0.00 & 31.00 & 0.00 & 0.00 \\
\hline$S 10$ & 33.00 & 0.08 & 0.29 & 33.00 & 0.10 & 0.18 \\
\hline$S 11$ & 29.00 & 0.00 & 0.00 & 29.00 & 0.00 & 0.00 \\
\hline$S 12$ & 33.00 & 0.09 & 0.39 & 33.00 & 0.16 & 0.21 \\
\hline$S 13$ & 17.00 & 0.00 & 0.00 & 17.00 & 0.00 & 0.00 \\
\hline$\overline{\mathrm{Avg}}$ & 31.00 & 0.06 & 0.20 & 29.77 & 0.09 & 0.12 \\
\hline
\end{tabular}

Table 4. Average results for 13 decision tables

\begin{tabular}{lrrrrrr}
\hline Threshold & \multicolumn{3}{c}{$G_{\alpha}$-algorithm } & \multicolumn{3}{c}{$L_{\alpha}$-algorithm } \\
$\alpha$ & $N_{A}$ & $m e_{A}^{G}$ & $m e_{A}^{L}$ & $N_{A}$ & $m e_{A}^{G}$ & $m e_{A}^{L}$ \\
\hline 13 & 12.7500 & 0.1325 & 0.2326 & 12.7500 & 0.1457 & 0.1883 \\
15 & 14.5000 & 0.1195 & 0.2467 & 14.2500 & 0.1357 & 0.1811 \\
17 & 17.0000 & 0.1091 & 0.2730 & 16.2308 & 0.1267 & 0.1734 \\
19 & 18.5385 & 0.1017 & 0.2402 & 17.6154 & 0.1222 & 0.1669 \\
21 & 20.3846 & 0.0937 & 0.2438 & 20.0769 & 0.1106 & 0.1555 \\
23 & 22.0769 & 0.0859 & 0.2309 & 21.7692 & 0.1093 & 0.1452 \\
25 & 24.2308 & 0.0791 & 0.2219 & 23.0000 & 0.1037 & 0.1414 \\
27 & 25.9231 & 0.0730 & 0.2192 & 23.9231 & 0.1005 & 0.1393 \\
29 & 27.3077 & 0.0685 & 0.2019 & 25.7692 & 0.0970 & 0.1338 \\
31 & 29.7692 & 0.0622 & 0.2089 & 28.2308 & 0.0915 & 0.1184 \\
33 & 31.0000 & 0.0576 & 0.1998 & 29.7692 & 0.0887 & 0.1168 \\
35 & 32.2308 & 0.0541 & 0.1851 & 30.8462 & 0.0762 & 0.1087 \\
\hline
\end{tabular}


Table 5. Changes of the average values of the parameters for the decrease of the threshold ('-' means decrease, otherwise increase)

\begin{tabular}{lrrrrrr}
\hline Threshold & \multicolumn{3}{c}{$G_{\alpha}$-algorithm } & \multicolumn{3}{c}{$L_{\alpha}$-algorithm } \\
$\alpha$ & $N_{A}$ & $m e_{A}^{G}$ & $m e_{A}^{L}$ & $N_{A}$ & $m e_{A}^{G}$ & $m e_{A}^{L}$ \\
\hline 13 & -1.7500 & 0.0130 & -0.0140 & -1.5000 & 0.0100 & 0.0072 \\
15 & -2.5000 & 0.0104 & -0.0264 & -1.9808 & 0.0091 & 0.0077 \\
17 & -1.5385 & 0.0074 & 0.0329 & -1.3846 & 0.0044 & 0.0065 \\
19 & -1.8462 & 0.0080 & -0.0037 & -2.4615 & 0.0116 & 0.0114 \\
21 & -1.6923 & 0.0078 & 0.0129 & -1.6923 & 0.0013 & 0.0103 \\
23 & -2.1538 & 0.0068 & 0.0091 & -1.2308 & 0.0056 & 0.0038 \\
25 & -1.6923 & 0.0061 & 0.0027 & -0.9231 & 0.0032 & 0.0021 \\
27 & -1.3846 & 0.0045 & 0.0172 & -1.8462 & 0.0035 & 0.0055 \\
29 & -2.4615 & 0.0063 & -0.0069 & -2.4615 & 0.0056 & 0.0154 \\
31 & -1.2308 & 0.0046 & 0.0091 & -1.5385 & 0.0027 & 0.0016 \\
33 & -1.2308 & 0.0035 & 0.0146 & -1.0769 & 0.0125 & 0.0081 \\
35 & - & - & - & - & - & - \\
\hline
\end{tabular}

increasing then again decreases.

- Let us consider the $m e_{A}^{G}$ parameter of the $L_{\alpha}$-algorithm. If we decrease the threshold, the average value in general is increasing and at the threshold 19, it seriously increases compared to the threshold 21.

- Let us consider the $m e_{A}^{L}$ parameter of the $L_{\alpha}$-algorithm. If we decrease the threshold, the average value in general increasing and at the thresholds 21 and 19 it seriously increases from the threshold 23.

The average values really depend on the nature of the considered decision tables as well as the size of the tables. Therefore it may be difficult to find a single threshold which can be considered as optimal one. But based on the above analysis, we may recommend the range 15 to 21 of thresholds that can be useful for building of decision trees for knowledge representation.

\section{Conclusions}

The goal of this paper is to understand which threshold of number of nodes should be chosen if we would like to minimize the number of nodes and misclassification of the decision trees at the same time. In this way, it is possible to obtain an understandable decision tree. For this, we designed two algorithms $G_{\alpha}$ and $L_{\alpha}$ to build binary split decision trees (BS-trees) having at most $\alpha$ vertices (where $\alpha$ varies between 13 to 35 with step 2). These algorithms are based on the bi-criteria optimization of $N$ and misclassification rate (either $m e^{G}$ or $m e^{L}$ ). We found an optimization model to adjust the parameters of decision trees and recommend a range of threshold 15 to 21 which can give us acceptable values of the parameters $m e^{G}$ and $m e^{L}$. In the future, it is possible to extend these algorithms to multi-label decision tables using BCO as explained in (Alsolami et al., 2020; Azad, 2018). Furthermore, we are also trying to invent new algorithms to restrict the number of branches in the DAG to reduce the time complexity of the algorithms. Moreover, in future, we are planning to extend bi-criteria optimization to the case of decision trees with hypothesis (Azad et al., 2021a,b,c,d).

\section{ACKNOWLEDGMENTS}

We are greatly indebted to Jouf University and King Abdullah University of Science \& Technology for the support of this research.

\section{References}

AbouEisha, H., Amin, T., Chikalov, I., Hussain, S. and Moshkov, M. (2019), Extensions of Dynamic Programming for Combinatorial Optimization and Data Mining, Vol. 146 of Intelligent Systems Reference Library, Springer.

Aldilaijan, A., Azad, M. and Moshkov, M. (2019), 'Experimental study of totally optimal decision trees', 
Fundam. Inform. 165(3-4), 245-261. URL:

https://doi.org/10.3233/FI-2019-1784

Almuallim, H., Kaneda, S. and Akiba, Y. (2002), 3 - development and applications of decision trees, in C. T. Leondes, ed., 'Expert Systems', Academic Press, Burlington, pp. 53-77.

Alsolami, F., Azad, M., Chikalov, I. and Moshkov, M. (2020), Decision and Inhibitory Trees and Rules for Decision Tables with Many-valued Decisions, Vol. 156 of Intelligent Systems Reference Library, Springer.

Azad, M. (2018), Decision and Inhibitory Trees for Decision Tables with Many-Valued Decisions, PhD thesis, King Abdullah University of Science \& Technology, Thuwal, Saudi Arabia.

URL: $h t t p: / / h d l . h a n d l e . n e t / 10754 / 628023$

Azad, M. (2020a), 'Decision trees with at most 19 vertices for knowledge representation', Trans. Rough Sets 22, 1-7.

URL: https://doi.org/10.1007/978-3-662-62798-3 $1_{-}$

Azad, M. (2020b), 'Knowledge representation using decision trees constructed based on binary splits', KSII Transactions on Internet and Information Systems 14(10), 4007-4024.

Azad, M., Chikalov, I., Hussain, S. and Moshkov, M. (2015), Multi-pruning of decision trees for knowledge representation and classification, in '3rd IAPR Asian Conference on Pattern Recognition, ACPR 2015, Kuala Lumpur, Malaysia, November 3-6, 2015’, IEEE, pp. 604-608.

Azad, M., Chikalov, I., Hussain, S. and Moshkov, M. (2018), Restricted multi-pruning of decision trees, in '13th International FLINS Conference on Data Science and Knowledge Engineering for Sensing Decision Support, FLINS', pp. 371-378.

Azad, M., Chikalov, I., Hussain, S. and Moshkov, M. (2021a), 'Entropy-based greedy algorithm for decision trees using hypotheses', Entropy 23(7).

URL: https://www.mdpi.com/1099-4300/23/7/808

Azad, M., Chikalov, I., Hussain, S. and Moshkov, M. (2021b), Minimizing depth of decision trees with hypotheses (to appear), in 'International Joint Conference on Rough Sets (IJCRS 2021), 19-24 September 2021, Bratislava, Slovakia'.

Azad, M., Chikalov, I., Hussain, S. and Moshkov, M. (2021c), Minimizing number of nodes in decision trees with hypotheses (to appear), in '25th International Conference on Knowledge-Based and Intelligent Information \& Engineering Systems (KES 2021), 8-10 September 2021, Szczecin, Poland'.

Azad, M., Chikalov, I., Hussain, S. and Moshkov, M. (2021d), 'Optimization of decision trees with hypotheses for knowledge representation', Electronics 10(13).

URL: $h t t p s: / / w w w . m d p i . c o m / 2079-9292 / 10 / 13 / 1580$

Azad, M., Chikalov, I. and Moshkov, M. (2019), Decision trees for knowledge representation, in K. Ropiak, L. Polkowski and P. Artiemjew, eds, '28th International Workshop on Concurrency, Specification and Programming, CS\&P 2019, Olsztyn, Poland, September 24-26, 2019', Vol. 2571 of CEUR Workshop Proceedings, CEUR-WS.org.

URL: http://ceur-ws.org/Vol-2571/CSP2019 paper 1.pdf

Azad, M., Chikalov, I., Moshkov, M. and Zielosko, B. (2013), 'A greedy algorithm for construction of decision trees for tables with many-valued decisions - A comparative study', Fundam. Inform.

128(1-2), 1-15.

URL: https://doi.org/10.3233/FI-2013-929 
Azad, M. and Moshkov, M. (2017), 'Multi-stage optimization of decision and inhibitory trees for decision tables with many-valued decisions', Eur. J. Oper. Res. 263(3), 910-921.

URL: https://doi.org/10.1016/j.ejor.2017.06.026

Bellman, R. (1954), 'The theory of dynamic programming', Bull. Amer. Math. Soc. 60(6), 503-515.

Boryczka, U. and Kozak, J. (2009), New algorithms for generation decision trees-ant-miner and its modifications, in A. Abraham, A. E. Hassanien, A. C. P. de Leon Ferreira de Carvalho and V. Snásel, eds, 'Foundations of Computational Intelligence - Volume 6: Data Mining', Vol. 206 of Studies in Computational Intelligence, Springer, pp. 229-262.

Breiman, L., Friedman, J. H., Olshen, R. A. and Stone, C. J. (1984), Classification and Regression Trees, Wadsworth and Brooks, Monterey, CA.

Chai, B.-B., Zhuang, X., Zhao, Y. and Sklansky, J. (1996), Binary linear decision tree with genetic algorithm, in 'Proceedings - International Conference on Pattern Recognition', Vol. 4, pp. 530-534.

Chang, K.-H. (2015), Chapter 5 - multiobjective optimization and advanced topics, in K.-H. Chang, ed., 'Design Theory and Methods Using CAD/CAE', Academic Press, Boston, pp. 325-406.

de Weck, O. (2004), 'Multiobjective optimization: History and promise', Invited Keynote Paper, GL2-2, the Third China-Japan-Korea Joint Symposium on Optimization of Structural and Mechanical Systems

2.

Dua, D. and Graff, C. (2017), 'UCI Machine Learning Repository. University of California, Irvine, School of Information and Computer Sciences'.

URL: http://archive.ics.uci.edu/ml

Ehrgott, M. and Gandibleux, X. (2000), 'A survey and annotated bibliography of multiobjective combinatorial optimization', OR Spectr. 22(4), 425-460.

Emmerich Michael T. M., D. A. H. (2018), 'A tutorial on multiobjective optimization: fundamentals and evolutionary methods', Natural Computing 17(3), 1585-609.

Garey, M. R. (1972), 'Optimal binary identification procedures', SIAM Journal on Applied Mathematics 23, 173-186.

Geiger, M. J. and Sevaux, M. (2011), The biobjective inventory routing problem - problem solution and decision support, in J. Pahl, T. Reiners and S. Voß, eds, 'Network Optimization', Springer Berlin Heidelberg, Berlin, Heidelberg, pp. 365-378.

Heath, D., Kasif, S. and Salzberg, S. (1993), 'Induction of oblique decision trees', Journal of Artificial Intelligence Research 2(2), 1-32.

Hopfe, C., Emmerich, M., Marijt, R. and Hensen, J. (2012), Robust multi-criteria design optimization in building design, in 'Proceedings of Building Simulation and Optimization', pp. 118-125.

Kouvelis, P. and Sayın, S. (2006), 'Algorithm robust for the bicriteria discrete optimization problem', Ann. Oper. Res. 147(1), 71-85.

Mehmood, Z., Gul, N., Altaf, M., Mahmood, T., Saba, T., Rehman, A. and Mahmood, M. (2018), 'Scene search based on the adapted triangular regions and soft clustering to improve the effectiveness of the visual-bag-of-words model', EURASIP Journal on Image and Video Processing 2018.

Moshkov, M. (2005), Time complexity of decision trees, in J. F. Peters and A. Skowron, eds, 'Trans. Rough Sets III', Vol. 3400 of Lecture Notes in Computer Science, Springer, pp. 244-459. 
Podgorelec, V., Kokol, P., Stiglic, B. and Rozman, I. (2002), 'Decision trees: An overview and their use in medicine', Journal of medical systems 26, 445-63.

Quinlan, J. (1986), 'Induction of decision trees', Mach Learn 1, 81-106.

Quinlan, J. R. (2014), C4. 5: programs for machine learning, Elsevier.

Rokach, L. and Maimon, O. (2008), Data Mining with Decision Trees: Theory and Applications, World Scientific Publishing, River Edge, NJ.

Rosenthal, S. and Borschbach, M. (2017), Design perspectives of an evolutionary process for multiobjective molecular optimization, in '9th International Conference on Evolutionary Multi-Criterion Optimization - Volume 10173', EMO 2017, Springer-Verlag, Berlin, Heidelberg, pp. 529-544.

Sarwar, A., Mehmood, Z., Saba, T., Qazi, K., Adnan, A. and Jamal, H. (2018), 'A novel method for content-based image retrieval to improve the effectiveness of the bag-of-words model using a support vector machine', Journal of Information Science $\mathbf{4 5}$.

Schumacher, H. and Sevcik, K. C. (1976), 'The synthetic approach to decision table conversion', Commun. ACM 19(6), 343-351.

Tian, J., Cheng, J. and Gong, Y. (2018), 'Optimization of the operating cost of sewage conveyance', Kuwait Journal of Science 45(2), 104-115.

van der Horst, E., Marques-Gallego, P., Mulder-Krieger, T., Veldhoven, J., Kruisselbrink, J., Aleman, A., Emmerich, M., Brussee, J., Bender, A. and Ijzerman, A. (2012), 'Multi-objective evolutionary design of adenosine receptor ligands', Journal of Chemical Information and Modeling 52(7), 1713-1721.

Wang, P., Emmerich, M., Li, R., Tang, K., Back, T. and Yao, X. (2015), 'Convex hull-based multiobjective genetic programming for maximizing receiver operating characteristic performance', IEEE Transactions on Evolutionary Computation 19(2), 188-200.

Yahyaoui, H., El-Qurna, J. and Almulla, M. (2020), 'Specification and recognition of service trust behaviors', Kuwait Journal of Science 47(1), 33-41.

Yevseyeva, I., Basto-Fernandes, V., Ruano-Ordas, D. and Mendez, J. R. (2013), 'Optimising anti-spam filters with evolutionary algorithms', Expert Systems with Applications 40(10), 4010 - 4021.

$\begin{array}{ll}\text { Submitted } & : 07 / 11 / 2020 \\ \text { Revised } & : 10 / 07 / 2021 \\ \text { Accepted } & : 10 / 07 / 2021 \\ \text { DOI } & : 10.48129 / \text { kjs. } 10725\end{array}$

